\title{
Stability and Performance of First-Order Linear Time-Delay Feedback Systems: An Eigenvalue Approach
}

\author{
Shu-An He and I-Kong Fong \\ Department of Electrical Engineering, National Taiwan University, Taipei 10617, Taiwan \\ Correspondence should be addressed to I-Kong Fong, ikfong@cc.ee.ntu.edu.tw
}

Received 25 April 2011; Revised 26 August 2011; Accepted 6 September 2011

Academic Editor: Onur Toker

Copyright $\odot 2011$ S.-A. He and I.-K. Fong. This is an open access article distributed under the Creative Commons Attribution License, which permits unrestricted use, distribution, and reproduction in any medium, provided the original work is properly cited.

\begin{abstract}
Linear time-delay systems with transcendental characteristic equations have infinitely many eigenvalues which are generally hard to compute completely. However, the spectrum of first-order linear time-delay systems can be analyzed with the Lambert function. This paper studies the stability and state feedback stabilization of first-order linear time-delay system in detail via the Lambert function. The main issues concerned are the rightmost eigenvalue locations, stability robustness with respect to delay time, and the response performance of the closed-loop system. Examples and simulations are presented to illustrate the analysis results.
\end{abstract}

\section{Introduction}

Systems with delays in internal signal transmissions are quite common in electrical, mechanical, biological, and chemical engineering problems. The delays may be inherent characteristics of system components or part of the control process [1]. The retarded type time-delay systems discussed in this paper belong to those that can be modeled by ordinary differential equations (ODEs) combined with difference terms in time, called the differential difference equations (DDEs) [2].

Recently, research works about the state solution, controllability, observability, and controller design for timedelay systems are very abundant $[3,4]$. In the field of controller design, many literature reviews [5-7] propose linear matrix inequality (LMI) conditions for finding controllers. However, the resultant LMI conditions are mostly sufficient only.

The retarded type time-delay systems are considered infinite dimensional and have transcendental characteristic equations with an infinite spectrum. Over the past decade, many works have been done to find the dominant eigenvalues or even the entire spectrum. For example, in [8] many approaches are introduced to iteratively find the approximate rightmost eigenvalues. Another important numerical method is proposed in [9], which uses the Lambert function to develop an expression for the spectrum. Subsequently, the expression is applied to decide the state solution, to discuss the controllability and observability, and to design state feedback controllers [10-13].

Through the Lambert function approach, theoretically the full view of spectrum can be observed. Recently, an auxiliary matrix is introduced to combine with the Lambert function for finding the spectrum of high-order time-delay systems, but it is also pointed out that the existence and uniqueness of such auxiliary matrix are still open problems [14]. Thus, as a basis for complex high-order time-delay systems, this paper discusses the first-order delay systems intensively through the Lambert function.

First-order linear systems with input delay have been focus of study in literature reviews such as $[8,15]$. To discuss stability, effects of three factors are of general concern: system parameter, controller gain, and delay size. In [15] P-control is studied, and only the range of stabilizing controller gain is analyzed via Padé approximation and crossing frequencies determination. In [8], a stability region diagram is constructed with respect to the above three factors, but the method suggested is iterative in nature and quite computationally intensive. Through the properties of Lambert function, this paper investigates the stability as well as performance of the first-order linear time delay systems with state feedback. Not only stability conditions are derived, but the entire closed-loop spectrum can be exposed, which 
facilitates the selection of controller gains to achieve the control objectives. More specifically, the rightmost eigenvalue will be located, and some deeper issues will be emphasized, including stabilization and stability robustness with respect to the delay time. Besides, how to select a feedback gain in order to obtain better response performance is also covered. Finally, examples and simulations are presented to illustrate the analysis results.

\section{Problem Formulation}

Consider the first-order time-delay system,

$$
\begin{gathered}
\dot{x}(t)=a_{0} x(t)+b u(t-h), \quad t \geq 0, \\
x(t)=x_{0}, \quad t=0,
\end{gathered}
$$

where $x \in \mathbb{R}$ is the state variable, $u \in \mathbb{R}$ is the input signal, $a_{0} \in \mathbb{R}$ and $b \in \mathbb{R}$ are the system parameters, $h$ is a nonnegative constant delay time, and $x_{0}$ is the initial condition. When a state feedback controller $u(t)=K x(t)$ is applied, (1) becomes

$$
\begin{gathered}
\dot{x}(t)=a_{0} x(t)+a_{1} x(t-h), \quad t \geq 0, \\
x(t)= \begin{cases}x_{0}, & t=0, \\
0, & t<0,\end{cases}
\end{gathered}
$$

where $a_{1}=b K$. The state-space model (2) is a DDE, for which stability and stabilizability are to be studied. The characteristic equation of (2) is $\Delta(s)=s-a_{0}-a_{1} e^{-s h}$, a transcendental one that has infinitely many roots. There are some numerical methods to solve this kind of equation, such as an ODE-based approach [16], but utilizing the Lambert function [17] exposes more properties of the eigenvalues.

\section{The Lambert Function}

The Lambert function $\mathcal{W}(\cdot)$ is the function that satisfies $\mathcal{W}(z) e^{\mathcal{W}(z)}=z, z \in \mathbb{C}$. It is a multivalued function, and the function values are classified as the principal branch and the $k$ th-branch for all non-zero integers $k$. Expressing $z=\alpha+j \beta$ and $\mathcal{W}(z)=\zeta+j \eta$ in the form of complex numbers, one has the relations

$$
\begin{aligned}
& \alpha=e^{\zeta}(\zeta \cos \eta-\eta \sin \eta), \\
& \beta=e^{\zeta}(\eta \cos \eta+\zeta \sin \eta) .
\end{aligned}
$$

In the next section, it will be clear that only $z \in \mathbb{R}$ needs to be discussed for the purpose of this paper, so either $\eta=$ 0 or $\zeta=-\eta \cot \eta$ with $\beta=0$, and $\mathcal{W}(z)=\zeta$ or $\mathcal{W}(z)=$ $-\eta \cot \eta+j \eta$. A partial plot of the function $\zeta=-\eta \cot \eta$ is displayed in Figure 1, and it is easy to infer the rest part of the function.

Based on Figure 1 and by convention, (4a) below defines the principal branch $\mathcal{W}_{0}(\mathrm{z})$ of the Lambert function, ( $\left.4 \mathrm{~b}\right)$

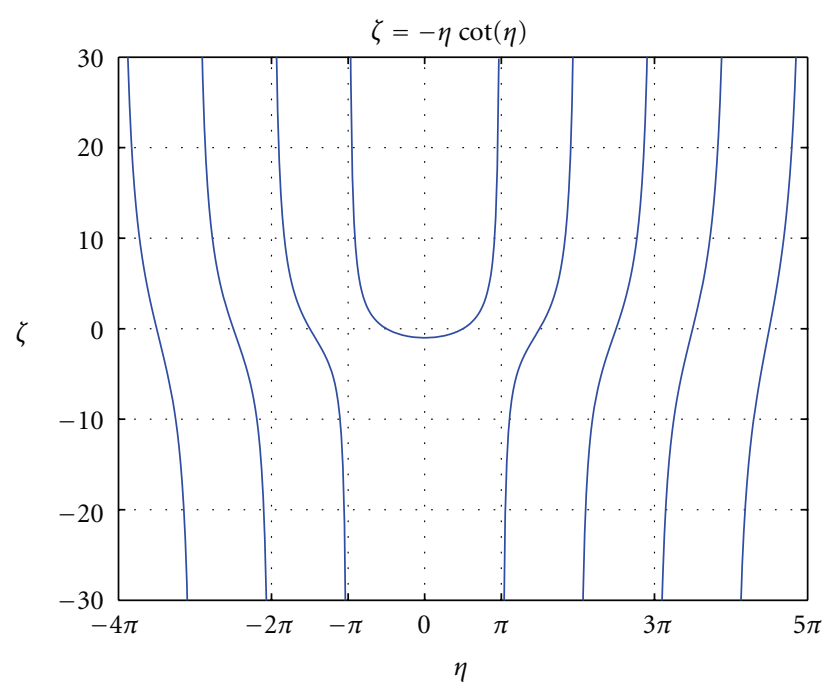

Figure 1: Plot of $\zeta=-\eta \cot \eta$.

defines the -1 st-branch $\mathcal{W}_{-1}(z)$, and (4c) defines the $k$ thbranch.

$$
\begin{gathered}
\mathcal{W}_{0}(z)= \begin{cases}-\eta \cot \eta+j \eta, & \text { for } \eta \in(0, \pi), \\
\zeta, & \text { for } \zeta \geq-1,\end{cases} \\
\mathcal{W}_{-1}(z)= \begin{cases}\zeta, & \text { for } \zeta<-1, \\
-\eta \cot \eta+j \eta, & \text { for } \eta \in(-2 \pi,-\pi) \cup(-\pi, 0),\end{cases}
\end{gathered}
$$

$$
W_{k}(z)=\left\{\begin{array}{l}
-\eta \cot \eta+j \eta, \\
\text { for } \eta \in((2 k-1) \pi, 2 k \pi) \cup(2 k \pi,(2 k+1) \pi), \\
-\eta \cot \eta+j \eta, \\
\text { for } \eta \in(2 k \pi,(2 k+1) \pi) \cup((2 k+1) \pi, 2(k+1) \pi), \\
k=-2,-3, \ldots
\end{array}\right.
$$

In Figure 2 a few branches of the Lambert functions are plotted, each with a different color, and the values of $z=$ $\alpha \in(-\infty, \infty)$ are labeled at corresponding positions with the black font. From Figure 2 many conclusions can be drawn, including the following two Lemmas.

Lemma 1. Only parts of $\mathcal{W}_{0}(z)$ and $\mathcal{W}_{-1}(z)$ are real-valued, and all other branches of the Lambert function are complexvalued.

In fact, only $\mathcal{W}_{0}(0)=0$, and all other branches are undefined at $z=0$. Also,

$$
\begin{gathered}
\mathcal{W}_{-1}(z)=\mathcal{W}_{0}^{*}(z), \quad \text { for } z \in\left(-\infty,-e^{-1}\right), \\
\mathcal{W}_{k}(z)=\mathcal{W}_{-k}^{*}(z), \quad \text { for } z \in(0, \infty), k=1,2, \ldots, \\
\mathcal{W}_{k}(z)=\mathcal{W}_{-(k+1)}^{*}(z), \quad \text { for } z \in(-\infty, 0), k=1,2, \ldots
\end{gathered}
$$

and $\mathcal{W}_{0}\left(-e^{-1}\right)=\mathcal{W}_{-1}\left(-e^{-1}\right)=-1$ 


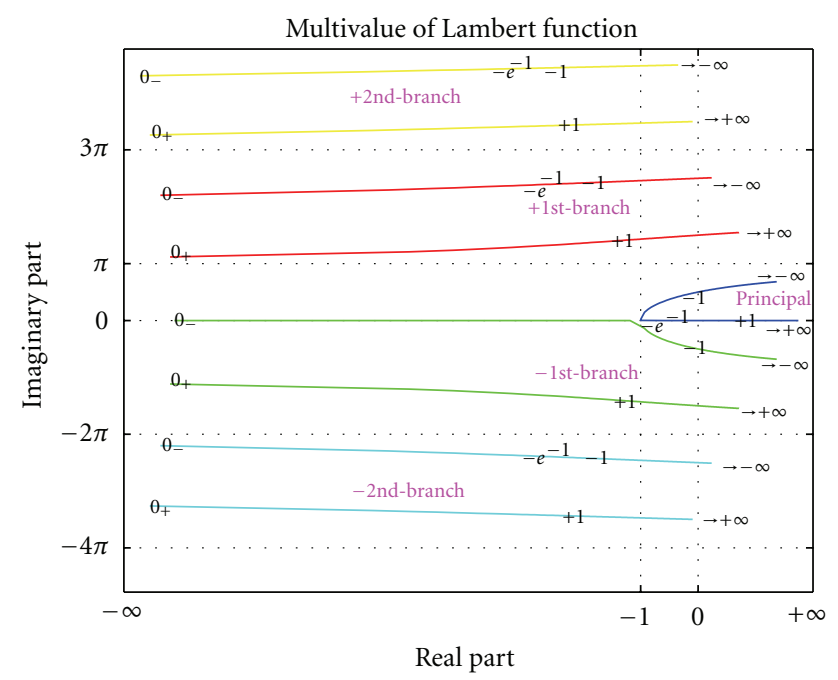

Figure 2: A few branches of the Lambert function parameterized by the values of $z=\alpha$.

Lemma 2. The largest lower bound of the real parts of the principal values is -1 .

Moreover, one has the following less intuitive Lemma.

Lemma 3. For any given $z \in \mathbb{R}$, the real part of $\mathcal{W}_{0}(z)$ is no less than that of $\mathcal{W}_{k}(z)$ for all $k \neq 0$.

Proof. Consider the three cases: $z \in\left(-\infty,-e^{-1}\right), z \in\left[-e^{-1}\right.$, $0)$, and $z \in(0, \infty)$ separately.

Case $1\left(z \in\left(-\infty,-e^{-1}\right)\right)$. In this case, all branches of the Lambert function are complex-valued. Given any $z \in \mathbb{R}$ and integer $k$, let $\mathcal{W}_{k}(z)=\zeta_{k}+j \eta_{k}$. Suppose that at $z=z_{0}$ and $z=z_{k}$ the principal branch and the $k$ th-branch values, respectively, have the same real part $\zeta_{0}=\zeta_{k}=\zeta$. Since in Figure 2, $z \rightarrow-\infty$ toward right in the branch segments with $z \in\left(-\infty,-e^{-1}\right)$, the proof will be completed if $z_{0} \geq$ $z_{k}$ is always true. Now $z_{0}-z_{k}=e^{\zeta}\left(\zeta \cos \eta_{0}-\eta_{0} \sin \eta_{0}-\right.$ $\left.\zeta \cos \eta_{k}+\eta_{k} \sin \eta_{k}\right)$ by $\mathcal{W}_{0}(z) e^{\mathcal{W}_{0}(z)}=\mathcal{W}_{k}(z) \mathrm{e}^{\mathcal{W}_{k}(z)}=z$, and $\eta_{k}=\eta_{0}+2 k \pi+\delta_{k}$ for all integer $k>0$ with some $\delta_{k}$. Since $e^{\zeta}>0$ and $\zeta_{k}=-\eta_{k} \cot \eta_{k}$, one has

$$
\begin{aligned}
\zeta\left(\cos \eta_{0}-\cos \eta_{k}\right)-\eta_{0} \sin \eta_{0}+\eta_{k} \sin \eta_{k} \\
=-\frac{\eta_{k}}{\sin \eta_{k}}\left(\cos \eta_{k} \cos \eta_{0}-\cos ^{2} \eta_{k}\right. \\
\left.\quad+\frac{\eta_{0}}{\eta_{k}} \sin \eta_{k} \sin \eta_{0}-\sin ^{2} \eta_{k}\right) \\
=-\frac{\eta_{k}}{\sin \eta_{k}}\left[\cos \left(\eta_{k}-\eta_{0}\right)-\sin \eta_{k} \sin \eta_{0}\right. \\
\left.\quad+\frac{\eta_{0}}{\eta_{k}} \sin \eta_{k} \sin \eta_{0}-1\right] \\
=-\frac{\eta_{k}}{\sin \eta_{k}}\left(\cos \delta_{k}-1\right)+\left(\eta_{k}-\eta_{0}\right) \sin \eta_{0} \\
\geq-\frac{\eta_{k}}{\sin \eta_{k}}\left(\cos \delta_{k}-1\right),
\end{aligned}
$$

where $\eta_{k} \in(2 k \pi, 2(k+1) \pi)$ and $\sin \eta_{k}>0$. Therefore, $z_{0} \geq$ $z_{k}$. For $k=-1$ and $k \leq-2,(5 \mathrm{a})$ and $(5 \mathrm{c})$, respectively, can be applied to extend the above result.

Case $2\left(z \in\left[-e^{-1}, 0\right)\right)$. In this case, the principal and the -1 st-branch are both real-valued, and from Figure 2 it can be seen that $\zeta_{0} \in[-1,0)$ and $\zeta_{-1} \in(-\infty,-1]$. Thus $\zeta_{0} \geq \zeta_{-1}$. For $k=1, \pm 2, \pm 3, \ldots, \eta_{0}=0$ implies

$$
\begin{aligned}
& e^{\zeta_{k}}\left(\zeta_{k} \cos \eta_{k}-\eta_{k} \sin \eta_{k}\right)=\zeta_{0} e^{\zeta_{0}} \\
& \Longrightarrow e^{\zeta_{k}}\left(-\eta_{k} \cot \eta_{k} \cos \eta_{k}-\eta_{k} \sin \eta_{k}\right)=\zeta_{0} e^{\zeta_{0}} \\
& \Longrightarrow-\eta_{k} e^{\zeta_{k}}=\zeta_{0} e^{\zeta_{0}} \sin \eta_{k} \\
& \Longrightarrow e^{\zeta_{0}-\zeta_{k}}=-\frac{\eta_{k}}{\zeta_{0} \sin \eta_{k}} .
\end{aligned}
$$

The last ratio of (7) is clearly no less than unity since $\eta_{k} /$ $\sin \eta_{k}$ is so and $\zeta_{0} \in[-1,0)$.

Case $3(z \in(0, \infty))$. In this case, all but the principal branch of the Lambert function are complex-valued. Again, suppose at $z=z_{0}$ and $z=z_{k}$, respectively, the principal branch and the $k$ th-branch values have the same real part $\zeta_{0}=\zeta_{k}=\zeta$. Note only non-negative $\zeta$ needs to be discussed in this case. Consequently, $\cos \eta_{k} \in(0,1)$, since here $\eta_{k} \in$ $((2 k-1) \pi, 2 k \pi)$ for $k>0$ and $\eta_{k} \in(2 k \pi,(2 k+1) \pi)$ for $k<0$, but with $\zeta_{k}=-\eta_{k} \cot \eta_{k} \geq 0$ the ranges of $\eta_{k}$ can be limited to $\eta_{k} \in((2 k-1 / 2) \pi, 2 k \pi)$ for $k>0$ and $\eta_{k} \in(2 k \pi,(2 k+1 / 2) \pi)$ for $k<0$. Because in Figure $2 z \rightarrow \infty$ toward right in the branch segments with $z \in(0, \infty)$, the proof will be completed if $z_{0} \leq z_{k}$ is always true. Now $z_{k}-z_{0}=e^{\zeta}\left(\zeta \cos \eta_{k}-\eta_{k} \sin \eta_{k}\right)-\zeta e^{\zeta}$ and

$$
\begin{aligned}
\zeta \cos \eta_{k}-\eta_{k} \sin \eta_{k}-\zeta & =\zeta\left(\cos \eta_{k}+\frac{\sin ^{2} \eta_{k}}{\cos \eta_{k}}-1\right) \\
& =\zeta\left(\frac{1}{\cos \eta_{k}}-1\right) .
\end{aligned}
$$

Hence the proof is completed.

\section{The Spectrum of First-Order Feedback Time-Delay Systems}

Consider the first-order time-delay system (2). Let $\lambda$ be an eigenvalue of (2). Then [9]

$$
\begin{aligned}
& \lambda-a_{0}-a_{1} e^{-\lambda h}=0 \\
& \Longleftrightarrow h\left(\lambda-a_{0}\right) e^{h\left(\lambda-a_{0}\right)}=a_{1} h e^{-a_{0} h} \\
& \Longleftrightarrow W\left(a_{1} h e^{-a_{0} h}\right)=h\left(\lambda-a_{0}\right) \\
& \Longleftrightarrow \lambda=\frac{1}{h} W\left(a_{1} h e^{-a_{0} h}\right)+a_{0} .
\end{aligned}
$$

In accordance with the Lambert function, $\lambda_{0}=a_{0}+$ $\mathcal{W}_{0}\left(a_{1} h e^{-a_{0} h}\right) / h$ is called the principal eigenvalue, and $\lambda_{k}=$ $a_{0}+W_{k}\left(a_{1} h e^{-a_{0} h}\right) / h, k= \pm 1, \pm 2, \ldots$, is called the $k$ th-branch 
eigenvalue. Note that the parameters $a_{0}, a_{1}$, and $h$ in the firstorder system are all real, so only $\beta=0$ is discussed for (3). By the above results there are some properties of the eigenvalues of (2) that can be obtained instantly.

(i) $\operatorname{Re}\left(\lambda_{0}\right) \geq \operatorname{Re}\left(\lambda_{\mathrm{k}}\right), k= \pm 1, \pm 2, \ldots$, for any given set of $a_{0}, a_{1}$ and $h$.

(ii)

$$
\begin{gathered}
a_{1} h e^{-a_{0} h}>-e^{-1} \Longleftrightarrow \lambda_{0} \in \mathbb{R}, \\
a_{1} h e^{-a_{0} h}=-e^{-1} \Longleftrightarrow \lambda_{0}=-\frac{1}{h}+a_{0}, \\
a_{1} h e^{-a_{0} h}<-e^{-1} \Longleftrightarrow \lambda_{0} \in \mathbb{C} .
\end{gathered}
$$

(iii) $-1 / h+a_{0} \leq \operatorname{Re}\left(\lambda_{0}\right)<\left|a_{0}\right|+\left|a_{1}\right|$.

(iv) $0 \leq \operatorname{Im}\left(\lambda_{0}\right)<\pi / h$.

Most of these properties are immediately implied by the results in Section 3. For the upper bound in property (iii), let $\lambda=\sigma+j \omega$, and from $\lambda=a_{0}+a_{1} e^{-\lambda h}$ one has $\sigma=$ $a_{0}+a_{1} e^{-\sigma h} \cos (-\omega h)$. Thus,

$$
|\sigma|=\left|a_{0}+a_{1} e^{-\sigma h} \cos (-\omega h)\right| \leq\left|a_{0}\right|+\left|a_{1}\right|\left|e^{-\sigma h}\right| .
$$

If $\sigma>0$ then $\operatorname{Re}\left(\lambda_{0}\right)<\left|a_{0}\right|+\left|a_{1}\right|$, and if $\sigma \leq 0$ then $\operatorname{Re}\left(\lambda_{0}\right) \leq$ 0 .

\section{Applications to Some Control Issues}

Without loss of generality, subsequently let $b$ be a given positive constant.

5.1. State Feedback for System Stabilization. For system (1) with the state feedback $u(t)=K x(t)$, property (ii) of Section 4 indicates that the principal eigenvalue of (2) is real-valued for $K \geq-e^{-1} /\left(b h e^{-a_{0} h}\right)$, and complex-valued for $K<-e^{-1} /\left(b h e^{-a_{0} h}\right)$. Moreover, by property (i) of Section 4 the principal eigenvalue has the largest real part among all eigenvalues. To stabilize system (2), one has to move the principal eigenvalue to the left half plane (LHP).

Theorem 4. There exists a state feedback gain $K$ such that system (2) can be asymptotically stabilized if and only if $a_{0}<$ $1 / h$.

Proof. Suppose $a_{0} \geq 1 / h$. Then property (iii) of Section 4 shows that $0 \leq \operatorname{Re}\left(\lambda_{0}\right)$ no matter what $K$ is. On the other hand, if $a_{0}<1 / h$, or $a_{0} h+\varepsilon=1$ for some $\varepsilon>0$, then in Figure 2 it can be seen that there is a $K$ such that

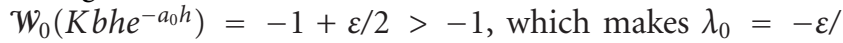
$(2 h)<0$.

Theorem 5. Suppose $a_{0}<1 / h$ in (1). The range of $K$ for the asymptotic stability of $(1)$ is $\left(-\eta_{0} /\left(b h \sin \eta_{0}\right),-a_{0} / b\right)$, where $a_{0} h=\eta_{0} \cot \eta_{0}$ and $\eta_{0} \in(0, \pi)$.

Proof. The values of $K$ for the asymptotic stability of (1) are those rendering the real part of $\lambda_{0}=a_{0}+W_{0}\left(K b h e^{-a_{0} h}\right) / h$

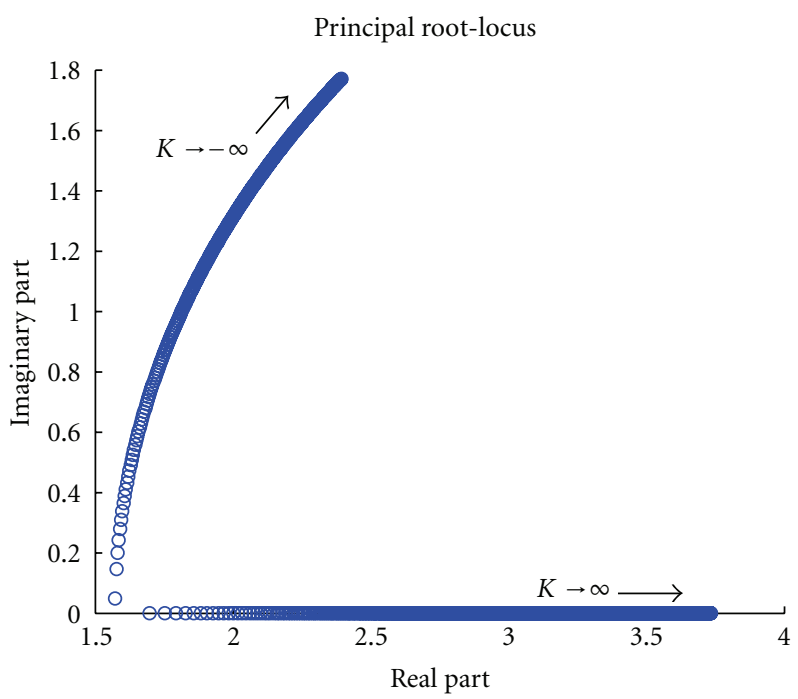

Figure 3: The principal eigenvalue locus versus $K$.

smaller than 0 , or that of $\mathcal{W}_{0}\left(K b h e^{-a_{0} h}\right)$ smaller than $-a_{0} h$. From Figure 2 and the assumption of $a_{0}<1 / h$ or $-1<$ $-a_{0} h$, a non-empty range of stabilizing gain $K$ exists, and the stabilizing gain $K$ must lie in the range of $\left(K_{1}, K_{2}\right)$, where $K_{2}$ makes $\zeta_{0}=-a_{0} h$ with

$$
K_{2} b h e^{-a_{0} h}=\zeta_{0} e^{\zeta_{0}}
$$

and $K_{1}$ makes $\zeta_{0}=-a_{0} h=-\eta_{0} \cot \eta_{0}$ with $\eta_{0} \in(0, \pi)$ and

$$
\begin{aligned}
K_{1} b h e^{-a_{0} h} & =e^{\zeta_{0}}\left(\zeta_{0} \cos \eta_{0}-\eta_{0} \sin \eta_{0}\right) \\
& =-e^{\zeta_{0}}\left(\eta_{0} \cot \eta_{0} \cos \eta_{0}+\eta_{0} \sin \eta_{0}\right) \\
& =-\frac{e^{\zeta_{0}} \eta_{0}}{\sin \eta_{0}} .
\end{aligned}
$$

Hence, $K_{1}=-\eta_{0} /\left(b h \sin \eta_{0}\right)$ and $K_{2}=-a_{0} / b$.

The result of Theorem 5 is known in [18], but here a different derivation is provided.

Definition 6. For a given $h>0$, the range of stabilizing gain $K$ is denoted as $\Theta_{h}$.

5.2. Robust Stability with Respect to Delay Size. The delay time $h$ in (1) is assumed to be a constant, but may be uncertain. In the practical applications, the delay time $h$ may not be estimated accurately. Therefore, it is important to analyze how robust the system (2) is with respect to the delay time $h$ under a selected stabilizing state feedback gain $K \neq 0$. Based on the signs of $a_{0}$ and $K$, four cases are discussed separately.

Case $1\left(a_{0} \leq 0\right.$ and $\left.K>0\right)$. Since $h$ is a positive delay time, the condition of Theorem 4 holds for all $a_{0} \leq 0$. Select a positive $K \in \Theta_{h^{*}}$, where $h^{*}$ is the assumed delay size. Thus $K \leq-a_{0} / b$ and the upper bound is independent of $h$. Also, by Theorem 5 the lower bound in $\Theta_{h}$ is nonpositive for all 


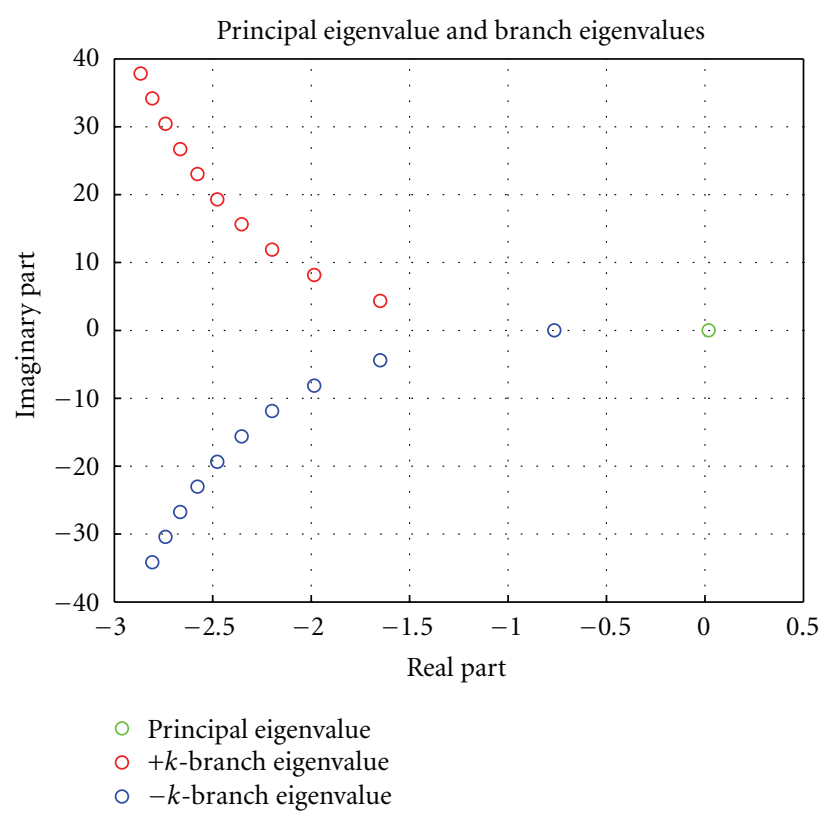

(a)

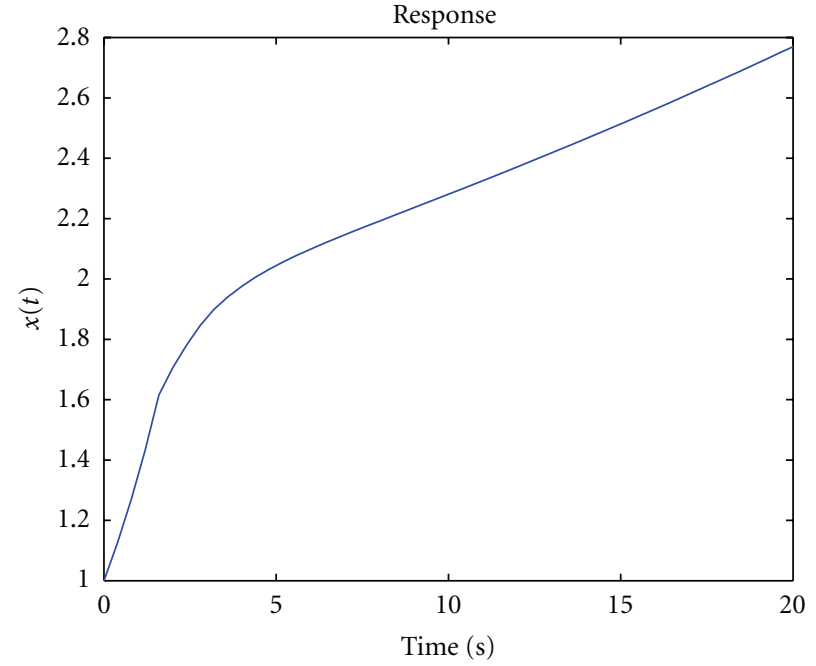

(b)

FIGURE 4: (a) The eigenvalues and (b) the response of system (2) with $K=-0.29$.

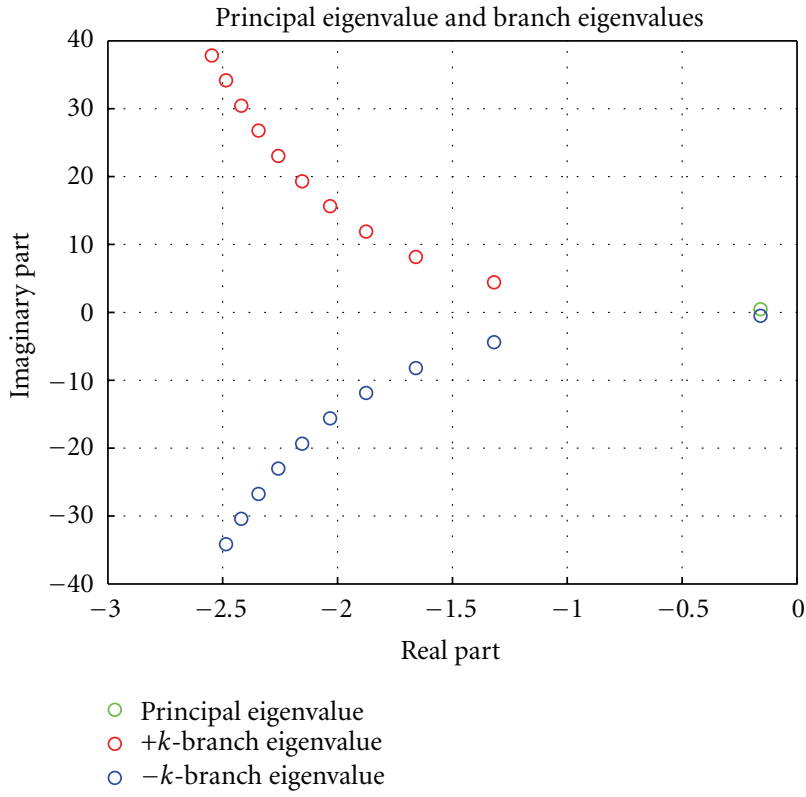

(a)

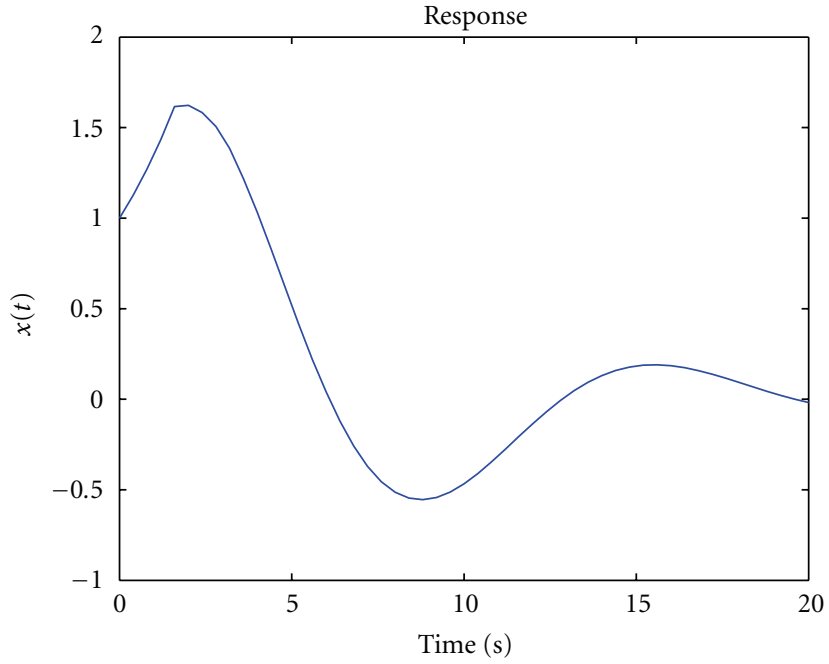

(b)

Figure 5: (a) The eigenvalues and (b) the response of system (2) with $K=-0.5$.

$h>0$. Hence the positive $K$ is in $\Theta_{h}$ for all $h>0$. In short, the feedback system is asymptotically stable independent of the delay time $h$.

Case $2\left(a_{0} \leq 0\right.$ and $\left.K<0\right)$. Suppose a negative $K \in \Theta_{h^{*}}$ is selected, where $h^{*}$ is the assumed delay size. Note that the principal eigenvalue is $\lambda_{0}=a_{0}+W_{0}\left(K b h e^{-a_{0} h}\right) / h$, so if $h<h^{*}$ then $K b h \mathrm{e}^{-a_{0} h}$ is less negative, and by observing the parameter values in the principal branch of Figure 2, it can be deduced that $\lambda_{0}$ will stay in the LHP. However, when $h-h^{*}$ is large enough, the real part of $\mathcal{W}_{0}\left(K b h e^{-a_{0} h}\right)$ will become positive. More specifically, as in the proof of Theorem 5, if $a_{0} h=\eta_{0} \cot \eta_{0}$ with some $\eta_{0} \in(\pi / 2, \pi)$ and $K=-\eta_{0} /\left(b h \sin \eta_{0}\right)=-a_{0} /\left(b \cos \eta_{0}\right)$, then $\lambda_{0}$ will move to the boundary of LHP. Note that such an $\eta_{0}$ exists if and only if $K \leq a_{0} / b$, which means if $K>a_{0} / b$ then the feedback system 


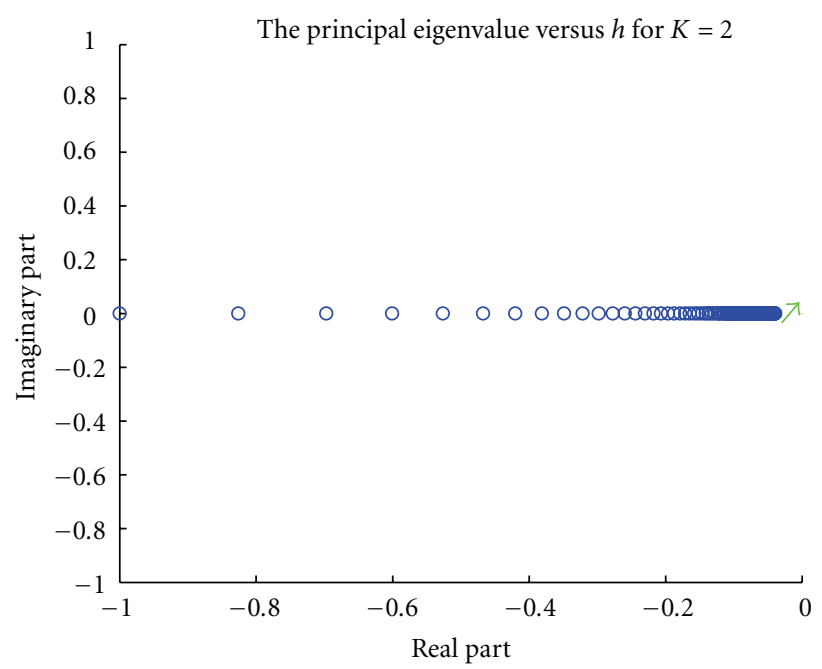

(a)

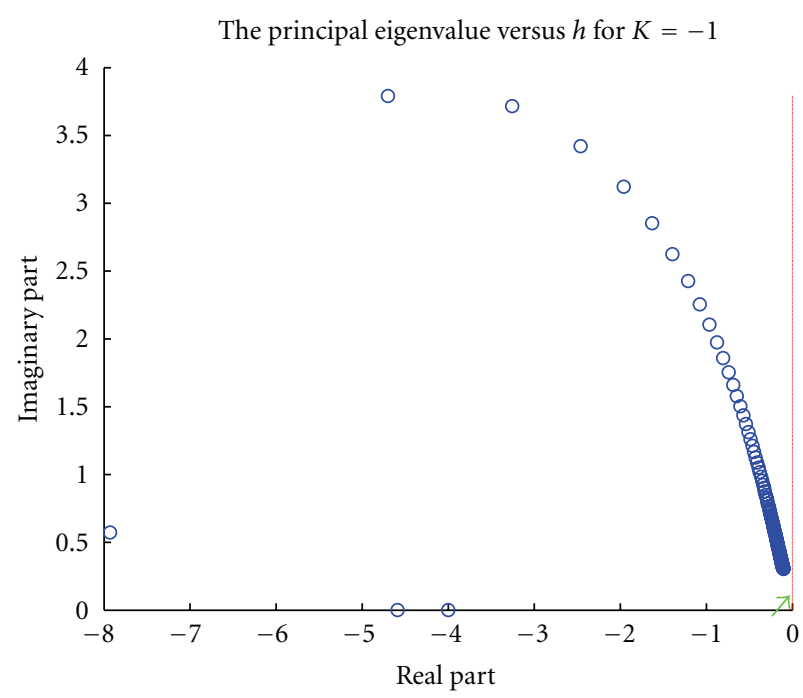

(c)

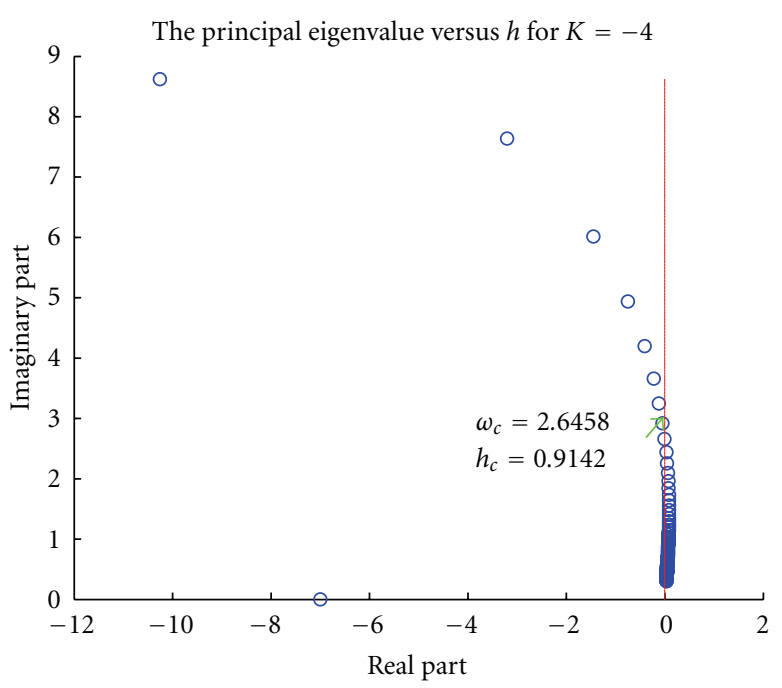

(b)

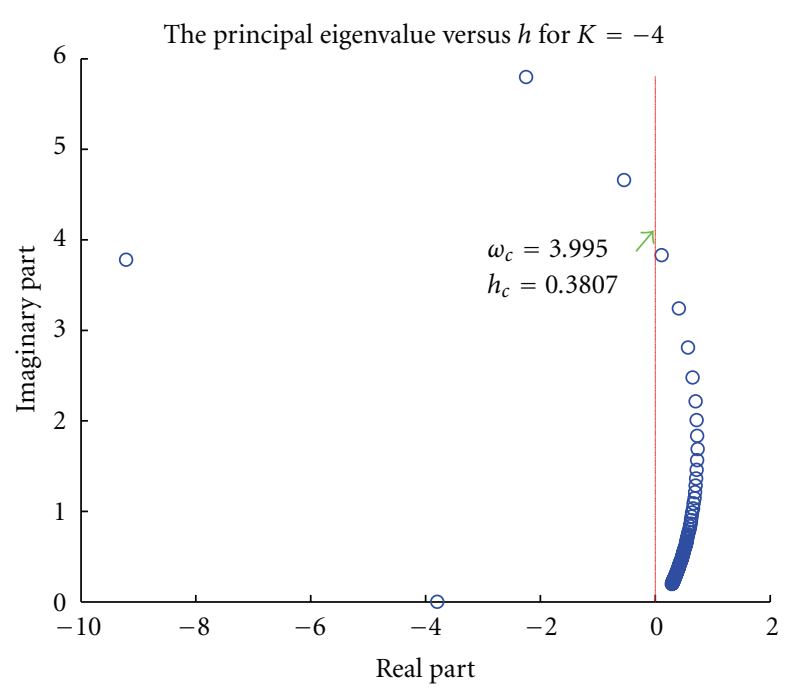

(d)

FIgure 6: The simulations for all cases in Section 5.2: (a) Case 1; (b) Case 2 (c); Case 2; (d) Case 3.

is stable independent of $h$, and if $K \leq a_{0} / b$ then stability will be lost for $h$ larger than $\left(\eta_{0} \cot \eta_{0}\right) / a_{0}$.

Case $3\left(a_{0}>0\right.$ and $\left.K<0\right)$. Again, suppose a negative $K \in \Theta_{h^{*}}$ is selected, where $h^{*}$ is the assumed delay size. Note that if $h<h^{*}$, then by Figure 1 the $\eta_{0}$ satisfying $a_{0} h=\eta_{0} \cot \eta_{0}$ is closer to $\pi / 2$ than the $\eta_{0}^{*}$ satisfying $a_{0} h^{*}=$ $\eta_{0}^{*} \cot \eta_{0}^{*}$. Therefore $\Theta_{h^{*}} \subseteq \Theta_{h}$ and the feedback system keeps asymptotic stability with the gain $K$. Next, if $h \geq h^{*}$ then $\Theta_{h} \subseteq \Theta_{h^{*}}$ and the feedback system keeps asymptotic stability if and only if $h$ is smaller than $\left(\eta_{0} \cot \eta_{0}\right) / a_{0}$, where $\eta_{0}=$ $\cos ^{-1}\left(-a_{0} /(b K)\right) \in(0, \pi / 2)$.

Case $4\left(a_{0}>0\right.$ and $\left.K>0\right)$. Since positive $K \notin \Theta_{h}$ for all $h$ when $a_{0}>0$, this case needs no discussion.

5.3. The Response Performance. Unlike non-delay systems, for which the system spectrum before and after state feedback stabilization have been thoroughly studied [19, 20], the corresponding problem for time-delay systems has not been probed deeply. Suppose it is desired to control the system (1) such that the response has a fast decay rate but is not oscillatory. In Theorem 5 , the range of $K$ for the asymptotic stability is decided, but not every $K$ in $\Theta_{h}$ results in a better response performance than that of the uncontrolled system. Actually, for positive $a_{0}$ all $K \in \Theta_{h}$ gives a better performance, but not necessarily so for negative $a_{0}$.

Theorem 7. For system (1), only $K \in\left(-\tilde{\eta}_{0} /\left(b h \sin \tilde{\eta}_{0}\right), 0\right)$, where $\tilde{\eta}_{0} \cot \tilde{\eta}_{0}=0$ and $\tilde{\eta}_{0} \in(0, \pi)$, gives a better response performance than that of the uncontrolled system.

Proof. Suppose for (1), $\Theta_{h}$ is not empty and $a_{0}<0$. From Figure 2, the set $\Theta_{h}$ consists of three subsets $\left(-\eta_{0} /\left(b h \sin \eta_{0}\right),-\tilde{\eta}_{0} /\left(b h \sin \tilde{\eta}_{0}\right)\right], \quad\left(-\tilde{\eta}_{0} /\left(b h \sin \tilde{\eta}_{0}\right), 0\right)$, and $\left[0,-a_{0} / b\right)$, where $a_{0} h=\eta_{0} \cot \eta_{0}, \tilde{\eta}_{0} \cot \tilde{\eta}_{0}=0$, and 


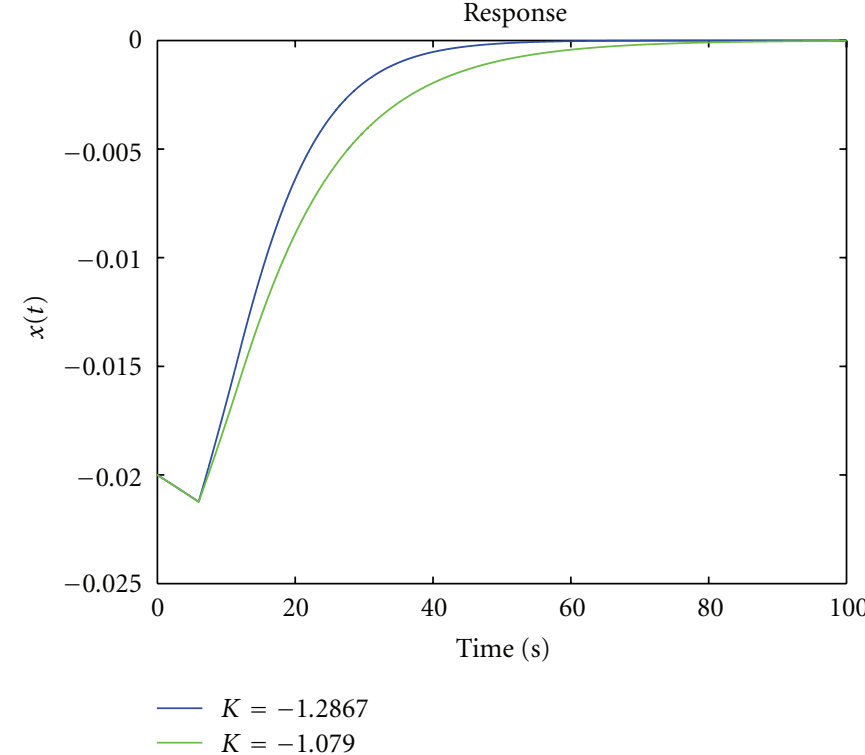

(a)

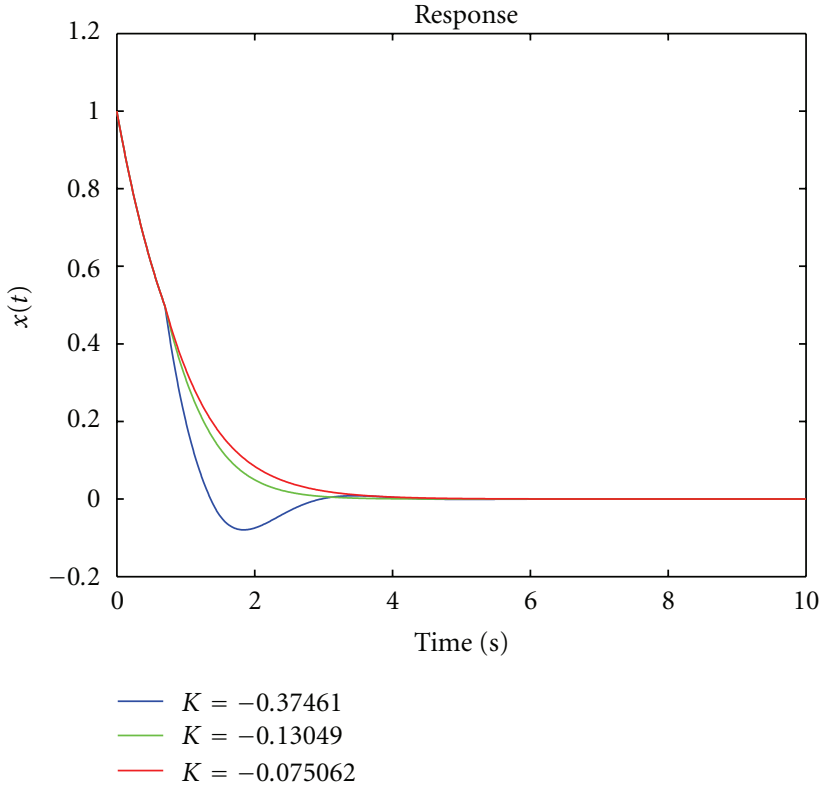

(b)

Figure 7: The responses of Example 10.

$\eta_{0}, \tilde{\eta}_{0} \in(0, \pi)$. However, only the $\lambda_{0}$ corresponding to $K \in$ $\left(-\tilde{\eta}_{0} /\left(b h \sin \tilde{\eta}_{0}\right), 0\right)$ has a more negative real part than $a_{0}$, which means such feedback gain results in a larger decay rate. Next, the set $\left(-\tilde{\eta}_{0} /\left(b h \sin \tilde{\eta}_{0}\right), 0\right)$ can be divided into $\left(-\tilde{\eta}_{0} /\left(b h \sin \tilde{\eta}_{0}\right),-e^{-1} /\left(b h e^{-a_{0} h}\right)\right)$ and $\left[-e^{-1} /\left(b h e^{-a_{0} h}\right), 0\right)$, where $\tilde{\eta}_{0} \cot \tilde{\eta}_{0}=0$ and $\tilde{\eta}_{0} \in(0, \pi)$. By property (ii), the $\lambda_{0}$ from $K \in\left(-\tilde{\eta}_{0} /\left(b h \sin \tilde{\eta}_{0}\right),-e^{-1} /\left(b h e^{-a_{0} h}\right)\right)$ implies an oscillatory response. Finally, if $K \in\left[-e^{-1} /\left(b h e^{-a_{0} h}\right), 0\right)$, then $\lambda_{0}$ is a real-valued. Although other $\lambda_{k}$ are complex-valued, $\lambda_{0}$ is the rightmost eigenvalue and far from others. Thus, oscillation only appears in the transient response. In fact, $K=-e^{-1} /\left(b h e^{-a_{0} h}\right)$ gives the largest decay rate and least oscillation.

\section{Examples and Simulations}

Example 8. Let the parameter combination $\left(a_{0}, b, K, h\right)=$ $(3,1, K, 0.7)$ be selected, where $a_{0}$ does not satisfy the condition of Theorem 4, so there is no stabilizing gain $K$. The principal eigenvalue locus versus $K \in(-\infty, \infty)$ is shown in Figure 3, and it can be seen that the principal eigenvalue always lies on the RHP no matter what $K$ is.

Then consider the parameter combination $\left(a_{0}, b, K, h\right)=$ $(0.3,1, K, 1.7)$, where $a_{0}<1 / h$ and there exists a feasible set $\Theta_{h}=\{K \mid K \in(-0.7424,-0.3)\}$. In Figure 4, a feedback gain $K \notin \Theta_{h}$ is used, so the principal eigenvalue locates on RHP and the response of $x(t)$ is divergent. In Figure 5, a feedback gain $K \in \Theta_{h}$ is used, so the principal eigenvalue locates on LHP and the response of $x(t)$ is convergent.

Example 9. In this example, a stabilizing $K$ is fixed to test the robustness of system (2) with respect to delay time $h$.
There are four simulations. The first one refers to Case 1 in Section 5.2, where $\left(a_{0}, b, K, h\right)=(-3,1,2, h)$, and the principal eigenvalue versus $h$ is shown as Figure 6(a). The stability of this system in this simulation is independent on $h$.

Then Figures 6(b) and 6(c) refer to Case 2 in Section 5.2, with $\left(a_{0}, b, K, h\right)=(-3,1,-4, h)$ and $(-3,1,-1, h)$, respectively. In Figure 6(b), the stability of this system is dependent on $h$, and the stability is maintained for $h \in[0,0.9142]$. In Figure 6(c), stability of this system is independent of $h$. The last simulation refers to Case 3 in Section 5.2 with $\left(a_{0}, b, K, h\right)=(0.2,1,-4, h)$. It is seen that the crossing frequency is 3.995, and the stability is maintained for $h=$ $[0,0.3807]$.

Example 10. Let the parameter combination $\left(a_{0}, b, K, h\right)=$ $(0.01,0.0506, K, 6)[21]$ be selected. The parameter $a_{0}$ is positive and satisfies Theorem 4 , and $\Theta_{h}=\{K \mid K \in$ $(-5.0488,-0.1976)\}$. In this example, all $K \in \Theta_{h}$ give the better closed-loop system response performance. For $K=-1.2867$ and $K=-1.079$, which correspond to $\lambda_{0}=-0.1567$ and $\lambda_{0}=-0.0763$, respectively, the responses are shown in Figure 7(a), where the blue line $\left(K=-e^{-1} /\left(b h e^{-a_{0} h}\right)\right)$ is better than green line in terms of decay rate. Consider another parameter combination $\left(a_{0}, b, K, h\right)=(-1,2, K, 0.7)$. The parameter $a_{0}$ is negative and satisfies Theorem 4 , and $\Theta_{h}=\{K \mid K \in$ $(-1.4600,-0.5000)\}$. For this case only $K \in(-1.122,0)$ produces a better closed-loop response performance. For three different gains $K=-0.37461, K=-0.13049$, and $K=-0.075062$, corresponding to $\lambda_{0}=-1.4 \pm 1.9558 j$, -2.4286 and -1.4 , respectively, the responses are shown in 
Figure 7(b), in which the blue and red lines have the same decay rate, but the blue line is oscillatory. Also, the green line is the best, since $K=-e^{-1} /\left(b h e^{-a_{0} h}\right)=-0.13049$.

\section{Conclusions}

Low-order time-delay systems are common in chemical engineering applications, and this paper studies the spectrum of first-order linear time-delay systems via the Lambert function. Stability and stabilization of such systems are discussed through the eigenvalue approach. By focusing on the principal eigenvalue, the intervals of stabilizing gains are obtained, and for a fixed stabilizing gain, the stability robustness of the system with respect to delay-time is explored. Moreover, through the full understanding of the spectrum, how to decide the feedback gain to obtain a better performance response is discussed. Three examples and simulations are shown to demonstrate the derived results.

Although this paper just focuses on the first-order system, the higher order systems can be discussed based on the analysis of this paper by using the partial fraction expansion approach. This will be the investigation goal of future works.

\section{Acknowledgment}

This research is supported by the National Science Council of the Republic of China under Grant NSC 98-2221-E-002148-MY3.

\section{References}

[1] K. Gu, V. L. Kharitonov, and J. Chen, Stability of Time-Delay Systems, Birkhäuser, Boston, Mass, USA, 2003.

[2] R. E. Bellman and K. L. Cooke, Differential-Difference Equations, Academic Press, New York, NY, USA, 1963.

[3] J. P. Richard, "Time-delay systems: an overview of some recent advances and open problems," Automatica, vol. 39, no. 10, pp. 1667-1694, 2003.

[4] S. A. He and I. K. Fong, "Time-delay effects on controllability in LTI systems," in Proceedings of the ICROS-SICE International Joint Conference (ICCAS-SICE '09), pp. 327-332, Fukuoka, Japan, August 2009.

[5] X. Li and C. E. de Souza, "Criteria for robust stability and stabilization of uncertain linear systems with state delay," Automatica, vol. 33, no. 9, pp. 1657-1662, 1997.

[6] E. Fridman and U. Shaked, "An improved stabilization method for linear time-delay systems," IEEE Transactions on Automatic Control, vol. 47, no. 11, pp. 1931-1937, 2002.

[7] W. H. Chen, Z. H. Guan, and X. Lu, "Delay-dependent robust stabilization and $H_{\infty}$-control of uncertain stochastic systems with time-varying delay," IMA Journal of Mathematical Control and Information, vol. 21, no. 3, pp. 345-358, 2004.

[8] W. Michiels and S. I. Niculescu, Stability and Stabilization of Time Delay Systems: An Eigenvalue Based Approach, Advances in Design and Control, SIAM, Philadelphia, Pa, USA, 2007.

[9] F. M. Asl and A. G. Ulsoy, "Analytical solution of a system of homogeneous delay differential equations via the Lambert function," in Proceeding of the American Control Conference, pp. 2496-2500, Chicago, Ill, USA, June 2000.

[10] Y. Chen and K. L. Moore, "Analytical stability bound for delayed second-order systems with repeating poles using
Lambert function W," Automatica, vol. 38, no. 5, pp. 891-895, 2002.

[11] C. Hwang and Y. C. Cheng, "A note on the use of the Lambert W function in the stability analysis of time-delay systems," Automatica, vol. 41, no. 11, pp. 1979-1985, 2005.

[12] E. Jarlebring and T. Damm, "The Lambert W function and the spectrum of some multidimensional time-delay systems," Automatica, vol. 43, no. 12, pp. 2124-2128, 2007.

[13] S. Yi, P. W. Nelson, and A. G. Ulsoy, "Analysis and control of time delayed systems via the Lambert W function," in Proceedings of the 17th IFAC World Congress, pp. 13414-13419, Seoul, Korea, 2008.

[14] S. Yi, A. G. Ulsoy, and P. W. Nelson, "Design of observer-based feedback control for time-delay systems with application to automotive powertrain control," Journal of the Franklin Institute, vol. 347, no. 1, pp. 358-376, 2010.

[15] G. J. Silva, A. Datta, and S. P. Bhattacharyya, PID Controllers for Time-Delay Systems, Birkhäuser, Boston, Mass, USA, 2004.

[16] A. B. Pitcher and R. M. Corless, "Quasipolynomial rootfinding: a numerical homotopy method," in Proceedings of the Canadian Undergraduate Mathematics Conference, Ontario, Canada, 2005.

[17] R. M. Corless, G. H. Gonnet, D. E. G. Hare, D. J. Jeffrey, and D. E. Knuth, "On the Lambert W function," Advances in Computational Mathematics, vol. 5, no. 4, pp. 329-359, 1996.

[18] V. Răsvan, "Delay independent and delay dependent Aizerman problem," in Unsolved Problems in Mathematical Systems and Control Theory, V. D. Blondel and A. Megretski, Eds., pp. 212220, Princeton University Press, Princeton, NJ, USA, 2004.

[19] W. J. Rugh, Linear System Theory, Prentice Hall, New York, NY, USA, 1996.

[20] C. T. Chen, Linear System Theory and Design, Oxford University Press, New York, NY, USA, 3rd edition, 1999.

[21] M. Shamsuzzoha and M. Lee, "Analytical design of enhanced PID filter controller for integrating and first order unstable processes with time delay," Chemical Engineering Science, vol. 63, no. 10, pp. 2717-2731, 2008. 

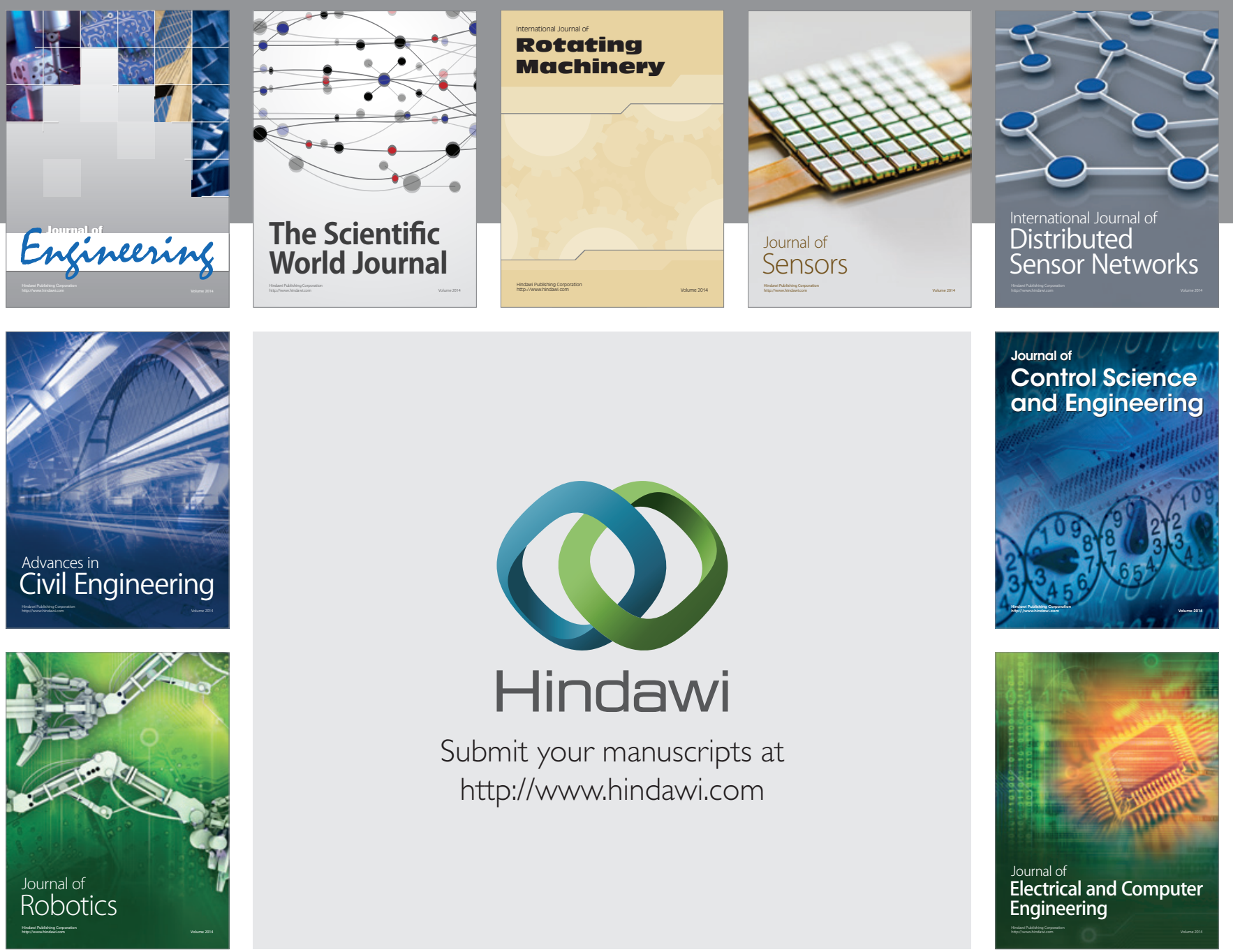

Submit your manuscripts at

http://www.hindawi.com
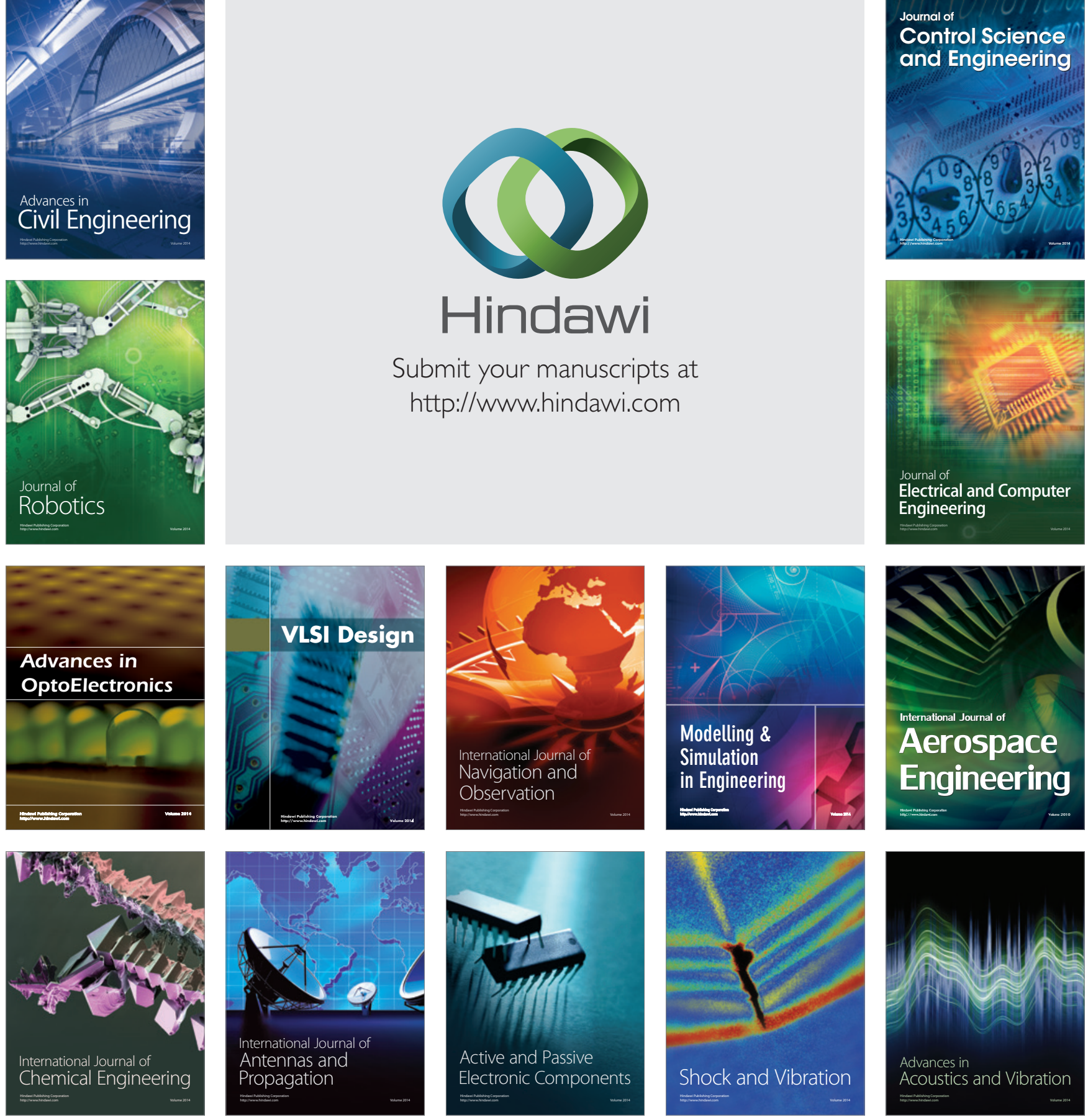